\title{
Retraction Note: Green urban garden landscape design and soil microbial environmental protection based on Virtual Visualization System
}

\author{
Lili Yu ${ }^{1} \cdot$ Xiling Xie $^{1} \cdot$ Lei Wei $^{2}$ \\ Published online: 6 December 2021 \\ (c) Saudi Society for Geosciences 2021
}

Retraction Note: Arabian Journal of Geosciences (2021) 14: 1155

https://doi.org/10.1007/s12517-021-07485-6

The Editor-in-Chief and the Publisher have retracted this article because the content of this article is nonsensical. The peer review process was not carried out in accordance with the Publisher's peer review policy. Authors Lili Yu and Xiling Xie have not responded to correspondence regarding this retraction. The Publisher has not been able to obtain a current email address for author Lei Wei.

The original article can be found online at https://doi.org/10.1007/ s12517-021-07485-6.

Lili Yu

yuli086@126.com

1 School of Art, Shandong Jianzhu University, Jinan 250101, Shandong, China

2 Jinan Hi-Tech Holding Group, Jinan 250101, Shandong, China 